\title{
Time to welcome the elderly into clinical trials
}

\author{
Michael E Farkouh and Valentin Fuster
}

As we apply evidence from randomized controlled trials (RCTs) to clinical practice, we are confronted with the issue of external validity or 'generalizability'. Do patients in clinical trials represent patients in the real world? Review of the literature indicates that there are misconceptions concerning age, ethnicity and sex, and how certain key subgroups respond to interventions compared with the general population. While much research has been devoted to evaluating evidence in women and ethnic minorities, very little work has focused on the elderly.

Subgroup analysis in RCTs can be problematic as special populations are almost always underrepresented. Despite these challenges, a great deal of effort has been devoted to evaluating whether African American patients respond differently to cardiovascular pharmacotherapies when compared with white patients. In fact, the interpatient variation in response to commonly prescribed antihypertensive medications is very similar in African American and white patients. The AHeFT trial demonstrated that African American patients with class II and IV heart failure benefit from combination therapy with isosorbide dinitrate and hydralazine; however, AHeFT enrolled only African American patients, the rationale for which is based on weak evidence. Perhaps other ethnic groups could have benefited from this combination therapy if studied in a similar protocol.

Similarly, when trials are analyzed on the basis of sex, no significant interactions emerge. A review of cardiovascular clinical trials published in leading journals indicated that only a quarter reported sex-specific results. Perhaps this figure is not too low after all. When reviewing the spectrum of cardiovascular diseases, no sex-related differences emerge; in the HOPE study, the benefit of ACE inhibitors in preserved left ventricular function was similar for men and women. In a meta-analysis of 10 RCTs of statins for primary and secondary prevention, the relative risk ratios were not significantly different for
Despite

advancing

age being

the strongest

predictor of cardiovascular

events, approximately $40 \%$ of all trials exclude the elderly.

ME Farkouh is the Director of the Mount Sinai Heart Clinical Trials Unit, Mount Sinai Cardiovascular Institute, NY, USA. $V$ Fuster is the Editorin-Chief of Nature Clinical Practice Cardiovascular Medicine.

\section{Competing interests} The authors declared no competing interests.

www.nature.com/clinicalpractice doi:10.1038/ncpcardio1387 women and men. Furthermore, the Antiplatelet Trialists' Collaboration evaluated 145 trials of aspirin and showed that women and men had similar reductions in vascular events.

Despite advancing age being the strongest predictor of cardiovascular events, approximately $40 \%$ of all trials exclude the elderly. Interestingly, industry-sponsored trials are more likely to exclude patients on the basis of age than those that are federally funded. In trials without an upper limit for age, participation of elderly patients is often low because of innate bias amongst physicians and patients that associates older age with inferior outcomes. Perhaps the best example of this bias relates to thrombolytic therapy for acute myocardial infarction. Although approximately $60 \%$ of deaths from myocardial infarction occur in patients $\geq 75$ years of age, only $10 \%$ of patients included in thrombolytic trials have been in this age group. Surprisingly to some, the relative risk reduction for mortality is comparable in the elderly and the young. Although risk of intracerebral hemorrhage is raised fourfold in the older populationa risk that had initially limited thrombolytics to younger patients - the net clinical benefit clearly favors a cost-effective reduction in mortality. Clearly, elderly patients are often at increased risk for complications from therapies; however, the risk-benefit balance should ultimately drive clinical decision making.

We desperately need more and better data on how to manage cardiovascular diseases in elderly patients, particularly in the advanced elderly population, the fastest-growing segment of the US population. Age should not be a valid criterion for trial exclusion. We believe it should become routine for eligible elderly patients to be approached to participate in cardiovascular RCTs. In fact, trials targeting the elderly population exclusively should also be encouraged. Patient care can only be enhanced by rigorous evaluation of potentially efficacious therapies in this important patient group. 